\title{
Superhydrophobic Surfaces Produced by Applying a Self- Assembled Monolayer to Silicon Micro/Nano-Textured Surfaces
}

\author{
Yong Song ${ }^{1}$, Rahul Premachandran Nair ${ }^{1}, \operatorname{Min} \mathrm{Zou}^{1}(\varangle)$, and Yongqiang Wang ${ }^{2}$ \\ ${ }^{1}$ Department of Mechanical Engineering, University of Arkansas, Fayetteville, AR 72701, USA \\ ${ }^{2}$ Ocean NanoTech, LLC., 2143 Worth Lane, Springdale, AR 72764, USA \\ Received: 8 September 2008 / Revised: 20 October 2008 / Accepted: 11 December 2008 \\ (C)Tsinghua University Press and Springer-Verlag 2009. This article is published with open access at Springerlink.com
}

\begin{abstract}
A novel way of producing superhydrophobic surfaces by applying a self-assembled monolayer (SAM) to silicon micro/nano-textured surfaces is presented in this paper. The micro/nano-textured surfaces on silicon substrates were generated by the aluminum-induced crystallization (AIC) of amorphous silicon (a-Si) technique. Octadecyltrichlorosilane (OTS) SAMs were then applied to the textured surfaces by dip coating. The topography and wetting properties of the resulting surfaces were characterized using scanning electron microscopy (SEM) and a video-based contact angle measurement system. The results show that by introducing OTS SAMs on the silicon micro/nano-textured surfaces, superhydrophobic surfaces with water contact angles (WCAs) of $155^{\circ}$ were obtained, as compared to the WCAs of OTS-modified smooth silicon surfaces of about $112^{\circ}$. Surface topography was found to directly influence the WCA as predicted by the Cassie-Baxter model.
\end{abstract}

\section{KEYWORDS}

Superhydrophobic, wetting property, micro/nano-textured surfaces, octadecyltrichlorosilane, self-assembled monolayer, aluminum-induced crystallization of amorphous silicon

\section{Introduction}

Superhydrophobic surfaces have attracted great attention in the past decade due to their important applications in surface self-cleaning, stiction prevention, and drag reduction [1-3]. The most well-known superhydrophobic surfaces are lotus leaves [4]. The surfaces of lotus leaves consist of micro-scale epidermal cells covered by nano-scale epicuticular wax crystals. The combination of surface roughness and wax coating renders the lotus leaves superhydrophobic with a water contact angle (WCA) of about $160^{\circ}$. Such water-repellent surfaces, along with very small sliding angles, enable lotus leaves to stay clean through self-cleaning (i.e., dust particles on the leaves can be easily removed by the water droplets that roll off the leaves).

Lotus leaf-like superhydrophobic surfaces have also been suggested to improve tribological performances of various devices such as microelectro-mechanical systems (MEMS) [5, 6]. It is well known that large adhesion and friction forces are major issues that impede the commercialization of MEMS devices consisting of moving parts [7, 8]. The current solutions to the tribological issues with MEMS are either to increase the surface

Address correspondence to mzou@uark.edu 
roughness through surface texturing or to improve the surface hydrophobicity through chemically modifying the silicon MEMS structure surfaces [7, 8]. Chemically modifying a smooth silicon surface can only lead to a hydrophobic surface with WCA of up to $120^{\circ}$ [9]. However, by combining surface chemical modification and topography modification, superhydrophobic surfaces with WCAs above $150^{\circ}$ can be achieved $[2,3]$, which enables further improvement of the tribological performance of MEMS devices.

Numerous methods of creating superhydrophobic surfaces have been reported by combining surface chemical and topography modifications, which include using aligned carbon nanotubes [10], electroless etching [11], electroplating [12], oxygen plasma etching [6], soft lithography imprinting [13], deep reactive ion etching [14], and many others. Each of these methods, however, has advantages and limitations. For example, some of them are not MEMS fabrication process compatible. Here we report an MEMS fabrication process compatible method that is easily implemented to generate superhydrophobic surfaces.

The superhydrophobic surfaces were fabricated by applying an octadecyltrichlorosilane (OTS) selfassembled monolayer (SAM) on silicon micro/nanotextured surfaces produced by the aluminuminduced crystallization (AIC) of amorphous $\mathrm{Si}(\mathrm{a}-\mathrm{Si}$ ) technique. OTS is one of the most extensively studied SAMs for adhesion and friction reduction in MEMS [15-17]. AIC of a-Si is an MEMS fabrication process compatible technique which has been studied extensively for production of large continuous polysilicon grains for electronic and photovoltaic applications [18-23]. For example, Nast et al. improved our fundamental understanding of the mechanism of AIC of a-Si by their discovery of the layer exchange phenomenon ocurring during the AIC of a-Si processes [18]. Schneider et al. investigated the influence of annealing temperature profiles on AIC of a-Si in order to improve the resulting silicon polycrystalline films [19]. Recently, Zou et al. reported the successful fabrication of silicon nanowires using AIC of a-Si [24]. Although both OTS and AIC of a-Si have been extensively studied individually, this is the first study combining OTS modification and AIC of a-Si to create superhydrophobic micro/nano-textured surfaces for potential applications in MEMS.

\section{Experimental}

\subsection{Fabrication of micro/nano-textured surfaces}

One-side polished p-type silicon wafers (100) were selected as substrates for producing micro/nanotextured surfaces using the AIC of a-Si technique. The silicon wafers were cleaned by acetone, isopropanol (IPA), and de-ionized (DI) water and then wet oxidized at $950{ }^{\circ} \mathrm{C}$ for $8 \mathrm{~h}$ to grow about $2 \mu \mathrm{m}$ thick silicon oxide films. The purpose of growing thick silicon oxide films prior to depositing a-Si is to prevent the crystalline structure of the substrates from affecting the AIC of a-Si process.

Amorphous silicon films of thickness ranging from $100 \mathrm{~nm}$ to $400 \mathrm{~nm}$ were then deposited on some of the wafers using plasma-enhanced chemical vapor deposition (PECVD). The RF power, chamber pressure, substrate temperature, and $\mathrm{SiH}_{4}$ flow rate were controlled at $20 \mathrm{~W}, 1 \mathrm{Torr}, 250{ }^{\circ} \mathrm{C}$, and $85 \mathrm{sccm}$, respectively. After a-Si deposition, the samples were removed from the PECVD system and exposed to air for three days to form a thin layer of native oxide on the top of the a-Si. The native oxide increases the consistency and repeatability of the AIC of a-Si results. These samples were then transferred to an evaporator for thermal deposition of an $800 \mathrm{~nm}$ thick aluminum layer.

The samples were then cut into small pieces of 1 in $x$ $1 \mathrm{in}$. and annealed in air in a conventional furnace at about $850{ }^{\circ} \mathrm{C}$ for $5 \mathrm{~s}$. After annealing, the excess $\mathrm{Al}$ was removed by wet selective etching, resulting in textured surfaces with micro/nano-structures. It should be emphasized that the annealing conditions were drastically different from those of traditional AIC of a-Si studies where the annealing temperatures are normally below $500{ }^{\circ} \mathrm{C}$, and the annealing durations are in hours [18-23].

\subsection{OTS SAM deposition}

The textured samples, along with thermal oxidecoated smooth silicon substrates, were then soaked 
in piranha solution $\left(\mathrm{H}_{2} \mathrm{O}_{2}: \mathrm{H}_{2} \mathrm{SO}_{4}=3: 7\right.$ by volume) to remove contaminants on the sample surfaces and to grow a fresh oxide layer on the substrate surfaces to facilitate assembly of OTS on the surfaces. After piranha cleaning, the samples were rinsed with DI water and toluene and blow-dried with $\mathrm{N}_{2}$ gas. The samples were then dipped into an OTS/ toluene solution to allow the OTS to uniformly selfassemble on the sample surfaces. Finally, the samples were rinsed with chloroform $\left(\mathrm{CHCl}_{3}\right)$, cleaned ultrasonically in chloroform, rinsed again with chloroform, and then dried in air.

\subsection{Sample characterizations}

Samples were characterized by scanning electron microscopy (SEM), energy dispersive X-ray spectroscopy (EDS), X-ray diffraction (XRD), and transmission electron microscopy (TEM) to obtain information on topography, elemental composition, and crystallinity of the textured features formed on the surfaces.

A video-based contact angle measurement system was used to measure the WCAs of the samples. The static WCAs of each sample were measured 5 times across the sample surface using the sessile drop method by dispensing $7 \mu \mathrm{L}$ drops of DI water on the sample surfaces. Sliding angles were measured by dropping a water droplet on a sample positioned on a tilting stage. The tilting angle of the stage was adjusted by a micrometer with a resolution of $0.03^{\circ}$. All WCA measurements were taken under ambient laboratory conditions with temperatures of about $20^{\circ} \mathrm{C}$ and about $45 \%$ relative humidity.

\section{Results and discussion}

\subsection{Micro/nano-textured surfaces}

The topographies of the samples after the AIC of a-Si process were characterized by SEM. SEM micrographs of different magnifications were taken across each sample to examine the micro-scale uniformity of the micro/nano-textures. Figures 1(a) and $1(b)$ show representative SEM micrographs of a smooth oxidized silicon sample before the AIC of a-Si process, and a nano-textured sample after the AIC of

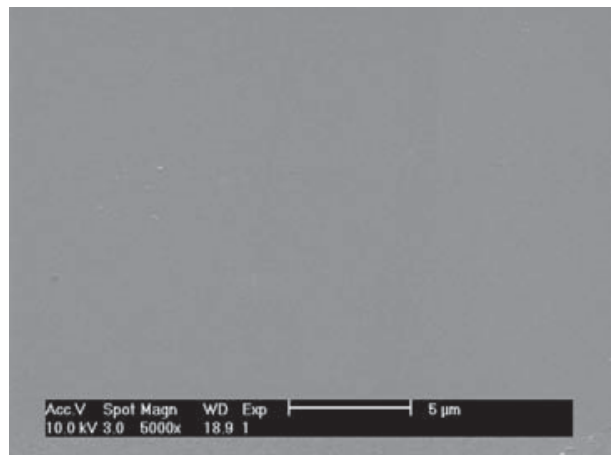

(a)

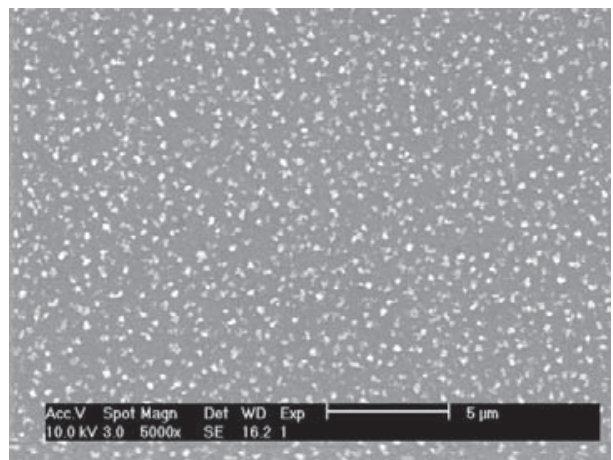

(b)

Figure 1 Representative SEM micrographs of (a) a smooth oxidized silicon sample before the AIC of a-Si process and (b) a micro/nanotextured sample after the AIC of a-Si process

a-Si process, respectively. Figure 1(b) shows that after the AIC of a-Si processes, the sample surface was textured by irregularly-shaped nano-scale islands with various sizes. Since the sample was thoroughly etched to remove $\mathrm{Al}$, these should not be $\mathrm{Al}$ islands.

EDS was used to investigate the elemental composition of the islands. Figure 2 shows a representative EDS spectrum taken from an island. The spectrum contains a large amount of $\mathrm{Si}$ and $\mathrm{O}$

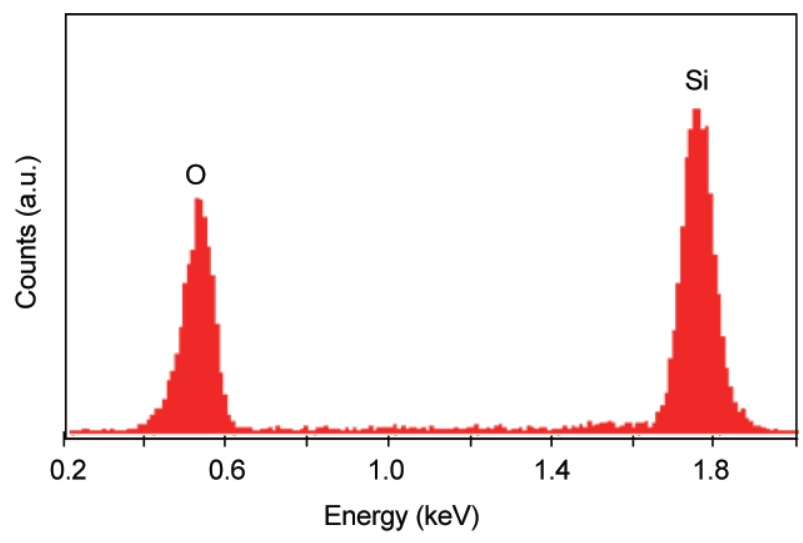

Figure 2 EDS spectrum taken from a nano-island. The spectrum shows a large amount of Si and $\mathrm{O}$ 
but no $\mathrm{Al}$, which indicates that these islands are made of silicon. The $\mathrm{O}$ signal probably comes from the thermal $\mathrm{SiO}_{2}$ film underneath the island and from the native $\mathrm{SiO}_{2}$ surrounding the island.

To determine whether the islands on the textured surface are crystalline silicon, XRD measurements were performed on both textured samples and a-Si coated silicon substrates. Although XRD cannot be performed on each individual island, it gives the spectrum of a collection of many islands. Figure 3 shows the XRD spectra of two sample surfaces: a textured sample surface and an a-Si-coated sample surface. The textured sample surface showed a strong peak around $28.5^{\circ}$, while the a-Si coated sample surface did not have any obvious peaks. The peak around $28.5^{\circ}$ indicates that the islands on the textured surface were crystalline silicon with (111) orientation.

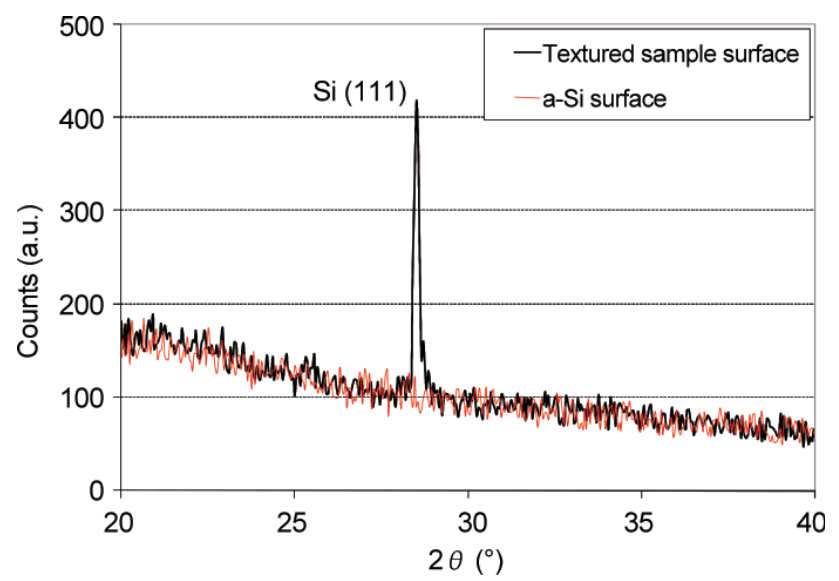

Figure 3 XRD spectrum of a textured sample and an a-Si-coated substrate. The peak around $28.5^{\circ}$ indicates that the islands on the textured surface are crystalline silicon with (111) orientation

Figure 4 is an electron diffraction pattern collected from an island that was detached from the substrate. The sample was prepared by scratching the crystallite from the substrate into an electron diffraction grid. The image shows both diffraction spots and rings, which indicates that the sample is a combination of polycrystalline and amorphous silicon. There are two possible explanations. One is that residual a-Si was transferred from the substrate to the TEM grid during TEM preparation. The other is that the islands are indeed a mixture of crystalline and amorphous silicon. This will be investigated in the future.



Figure 4 Electron diffraction pattern taken from a silicon nanoisland showing diffraction spots from crystalline material and rings from amorphous material. This indicates the nano-island is a polysilicon nanocrystallite

\subsection{Optimization of OTS deposition conditions}

Although the application of OTS SAMs on smooth silicon surfaces has been studied extensively [25, 26], the application of an OTS SAM on micro/nanotextured silicon surfaces has not been reported before. Therefore, we performed three experiments in order to find the most suitable conditions for applying the OTS SAMs on the textured surfaces, including finding the appropriate cleaning conditions for the substrates, deciding the OTS reaction time, and optimizing the OTS concentration.

Three substrate cleaning conditions were studied for smooth Si samples: (1) soaking samples in piranha solution at $90{ }^{\circ} \mathrm{C}$ for $30 \mathrm{~min}$; (2) soaking samples in piranha solution at $20^{\circ} \mathrm{C}$ for $1 \mathrm{~h}$; (3) soaking samples in piranha solution at $20{ }^{\circ} \mathrm{C}$ for $2 \mathrm{~h}$. The results showed that samples processed under the first conditions had the highest average WCA of $111.2^{\circ}$, but it was only $1.4^{\circ}$ higher than that of samples processed under the second conditions $\left(109.8^{\circ}\right)$. Since piranha solution is dangerous to use when heated, the increase in WCA when using piranha solution at $90{ }^{\circ} \mathrm{C}$ cannot be justified on safety grounds. Samples processed under the second and third conditions had similar WCAs $\left(109.8^{\circ}\right.$ and $110.1^{\circ}$ ), which are close to the reported WCAs of smooth Si surface modified with OTS SAMs [25]. Since the second condition took less processing time, it was chosen as the substrate cleaning condition for subsequent experiments. 
Two OTS SAM-reaction times were studied on both smooth and textured silicon samples: (1) $10 \mathrm{~min}$ and (2) $1 \mathrm{~h}$. The selection of these two-reaction times is based on the growth kinetics of OTS SAMs on $\mathrm{Si}(100) / \mathrm{SiO}_{2}$ surfaces. It has been reported that the initial adsorption of an OTS SAM on a silicon substrate is very fast. A full surface coverage can be reached in approximately 1 min, depending on the OTS concentration [25]. Longer reaction time only increase the WCA by a few degrees. We chose $10 \mathrm{~min}$ as one reaction time to allow sufficient time for the maximum WCA to be reached and $1 \mathrm{~h}$ as the other reaction time to see if there is any change on extending the reaction time further. Six textured and two control samples were used in this study, four for each reaction time. The WCAs of the smooth samples with $10 \mathrm{~min}$ and $1 \mathrm{~h}$ reaction times were $111^{\circ}$ and $114^{\circ}$, respectively. All three textured samples dipped for $10 \mathrm{~min}$ were superhydrophobic, but only one of the three textured samples dipped for $1 \mathrm{~h}$ showed superhydrophobicity. Therefore, a reaction time of 10 min was chosen for subsequent experiments.

Two OTS/toluene solution mass concentrations (OTS : toluene) were studied: (1) $0.1 \%$ and (2) $1 \%$. Six textured and two control samples were used in this study, four for each concentration. The WCAs of the smooth samples dipped in $0.1 \%$ and $1 \%$ solutions were $111.2^{\circ}$ and $111.7^{\circ}$, respectively. The WCAs of the three textured samples produced with $0.1 \%$ concentrations were $141.5^{\circ}, 141.7^{\circ}$, and $137.4^{\circ}$, with an average of $140.2^{\circ}$. The WCAs of the three textured samples produced with $1 \%$ concentrations were $140.2^{\circ}$, $141.2^{\circ}$, and $141.1^{\circ}$, with an average of $140.8^{\circ}$. Because the WCAs of the samples dipped in $1 \%$ solution are more reproducible than those dipped in $0.1 \%$ solution, $1 \%$ was chosen as the OTS concentration for subsequent experiments.

\subsection{Wetting properties of the textured surfaces}

The effects of surface texturing on the wetting properties were studied by comparing the WCAs of the textured surfaces with the WCAs of the smooth Si surfaces before and after OTS SAM modifications. The optimal conditions determined above were used when applying the OTS SAM to the surfaces. Figures 5(a) and $5(\mathrm{~b})$ show that OTS modification of a smooth silicon surface increased its WCA from $46^{\circ}$ to $112^{\circ}$, which matches the WCA of a full coverage of OTS SAM on a smooth Si surface reported in Ref. [25]. Figures 5(c) and 5(d) show no detectable surface topography changes before and after the OTS selfassembly on the surface, suggesting uniform OTS assembly on the surface.

Figures 6 (a) and 6(b) show that the WCA of a textured silicon sample was $37^{\circ}$, but after coating with OTS SAM it was increased to $155^{\circ}$, which is in the superhydrophobic range. The measured sliding angle of this superhydrophobic sample was smaller than $1^{\circ}$. The much higher WCA of the OTS SAM modified textured silicon surface compared with that of the OTS SAM modified smooth silicon surface suggests the importance of surface texturing in improving the surface hydrophobicity. Figures 6(c) and $6(\mathrm{~d})$ also show no detectable surface topography

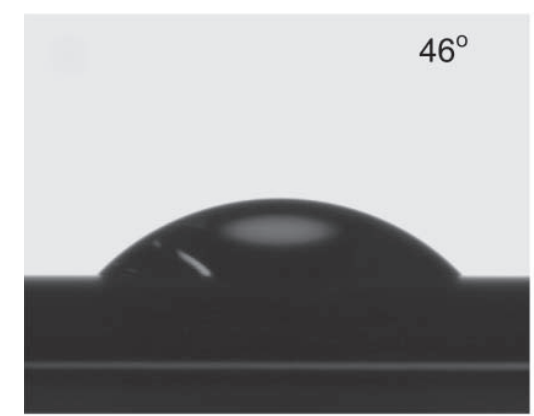

(a)

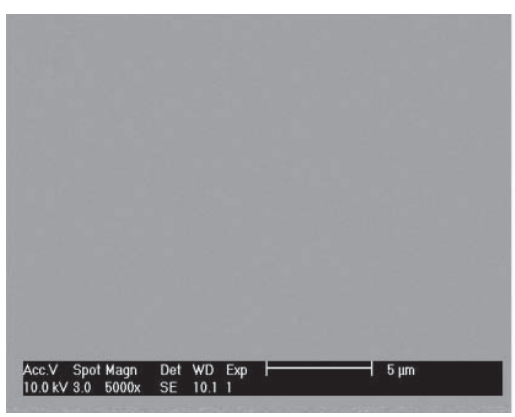

(c)

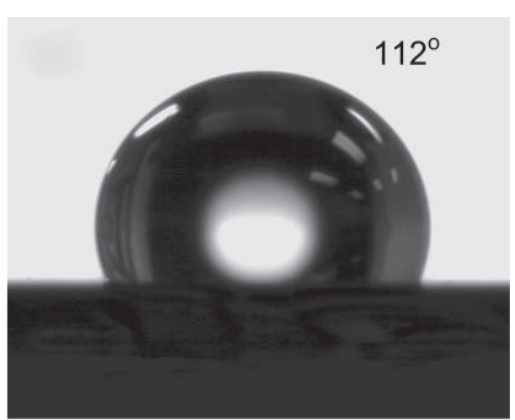

(b)

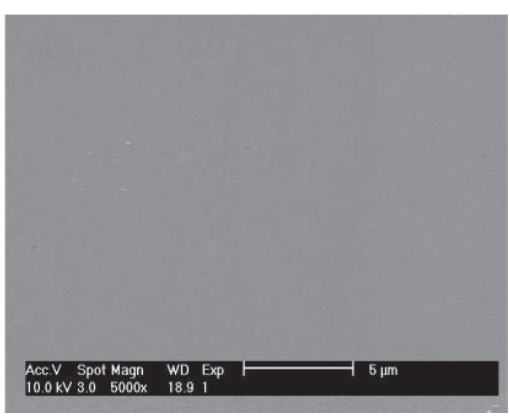

(d)
Figure 5 WCA pictures of a smooth Si sample (a) before OTS deposition, and (b) after OTS deposition, and SEM micrographs of the smooth Si surface (c) before OTS deposition, and (d) after OTS deposition 


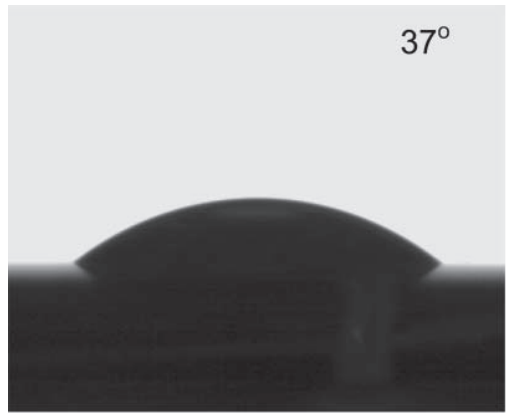

(a)



(c)

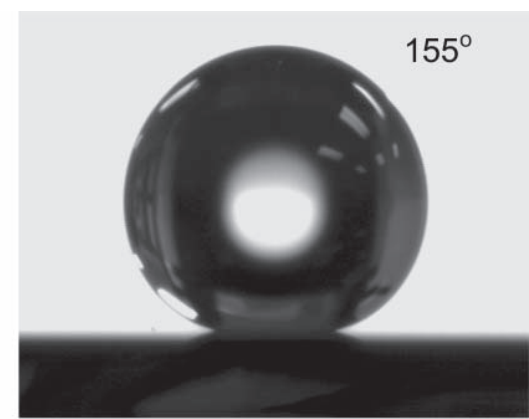

(b)

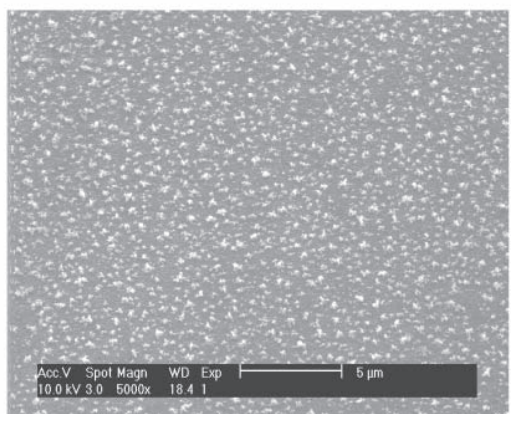

(d)
Figure 6 WCA pictures of a textured sample (a) before OTS deposition, and (b) after OTS deposition, and SEM micrographs of the textured sample (c) before OTS deposition, and (d) after OTS deposition

changes before and after the OTS self-assembly on the surface, suggesting uniform OTS assembly on the surface.

The superhydrophobicity of the OTS SAM modified textured Si surfaces is due to both chemical and topographic factors. As for the former, before OTS self-assembly, the terminal groups of the oxidized Si surface were $\mathrm{O}$ atoms, which are hydrophilic by nature. When the OTS self-assembly was complete, the hydrophilic $\mathrm{O}$ atoms were no longer available and the surface became hydrophobic because of the hydrophobic alkyl chain of the OTS. As for topography, air was trapped between the textured features, which prevents the the water droplet from wetting the surface and thus improves the hydrophobicity of the surface.

Because surface topography has significant effects on the surface wetting properties, as can be seen from a comparison of Figs. 5 and 6, such effects were further studied by investigating the wetting properties of various topographies. In this study, three groups of samples A, B, and C with different topographies were produced by changing the a-Si thickness and annealing conditions. Samples from group A were produced with $400 \mathrm{~nm}$ a-Si, annealed at $800{ }^{\circ} \mathrm{C}$ for $15 \mathrm{~s}$; samples from group B were produced with $200 \mathrm{~nm}$ a-Si, annealed at $800{ }^{\circ} \mathrm{C}$ for $20 \mathrm{~s}$; and samples from group $\mathrm{C}$ were produced with $300 \mathrm{~nm}$ a-Si, annealed at $750{ }^{\circ} \mathrm{C}$ for $10 \mathrm{~s}$. The reason for selecting these process parameters is that they produced three distinct topographies. Figures 7(a)-7(c) show representative SEM micrographs of the micro/nano-textured surfaces from groups A, B, and C, respectively, after OTS SAM modification. Figure 7 (a) shows that the surface of the sample A was covered by extruded networks of large silicon grains with a small number of grain boundaries extruding further from the surface. Figure 7(b) shows that the surface of sample B was covered by isolated polycrystalline silicon islands, instead of by a polycrystalline silicon network. The microsized silicon crystallites are randomly distributed on the surface, forming a micro-textured silicon surface. Figure 7(c) shows that the sizes of silicon crystallites in sample $C$ are significantly smaller than those in sample B (Fig. 7(b)). These randomly distributed nano-sized crystallites form a nano-textured surface. The WCAs of these three topographies are very different. The measured WCAs of the OTS modified textured samples are $133^{\circ}, 143^{\circ}$, and $155^{\circ}$ for the samples with silicon grain network (A), microsized textured features (B), and nano-sized textured features (C), respectively. Even though these surfaces have different WCA values, they are all much larger than that of the OTS modified smooth $\mathrm{Si}\left(112^{\circ}\right)$.

The impact of surface texture on surface WCAs can be explained by the Cassie-Baxter model [27] that describes the wetting of rough surfaces. The CassieBaxter model assumes that a water droplet contacts the top of a rough surface with air trapped in between the rough asperities on the surface. Therefore, the droplet contacts both the rough asperities and the air in between the asperities. In this case, the apparent 


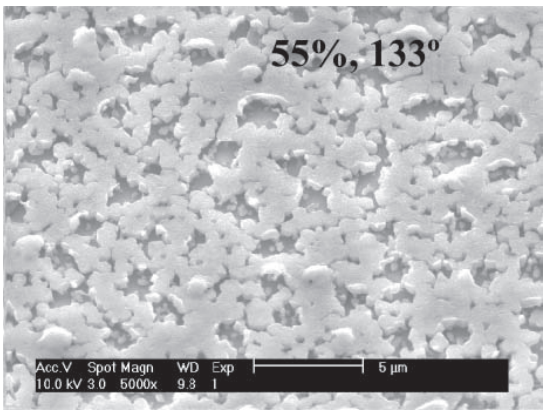

(a)

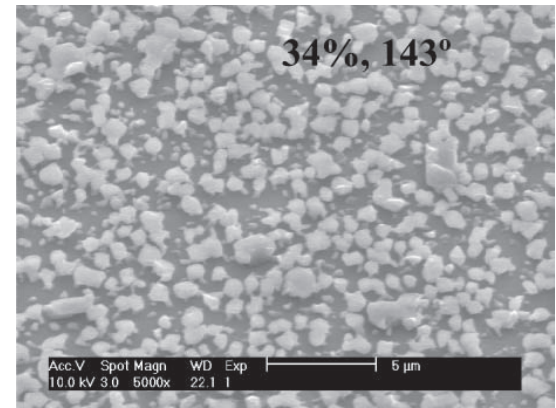

(b)

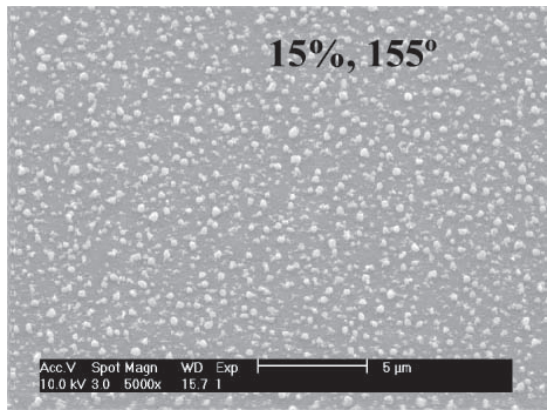

(c)

Figure 7 SEM micrographs of the samples with three different topographies: (a) $50 \%$ of the surface is covered by textured features and the WCA is $133^{\circ}$; (b) $34 \%$ of the surface is covered by textured features and the WCA is $143^{\circ}$; (c) $15 \%$ of the surface is covered by textured features and the WCA is $155^{\circ}$

WCA, $\theta^{*}$, is given by

$$
\begin{aligned}
& \cos \theta^{*}=f_{1} \cos \theta-f_{2} \\
& f_{1}+f_{2}=1
\end{aligned}
$$

where $f_{1}$ is the surface area fraction of the solid and $f_{2}$ is the surface area fraction composed of voids filled with air. In this study, values of $f_{1}$ were calculated using ImageJ developed by the National Institute of Health. It was found that the values of $f_{1}$ for the samples from groups A, B, and C are $55 \%, 34 \%$, and $15 \%$, respectively. Applying these values and the WCA value of the OTS SAM modified smooth silicon surface of $112^{\circ}$ to Eqs. (1) and (2), we calculate the WCAs of the SAM modified micro/nano-textured silicon surfaces to be $131^{\circ}, 142^{\circ}$, and $155^{\circ}$ for the samples shown in Figs. 7(a)-7(c), respectively. The predicted WCAs are in very good agreement with the measured WCAs.

\section{Conclusions}

In summary, this work explores the utilization of a combination of OTC and AIC of a-Si to fabricate superhydrophobic surfaces. It was shown that this novel technique can significantly improve the hydrophobicity of a silicon surface, resulting in superhydrophobic surfaces with a WCA of $155^{\circ}$ and a sliding angle of less than $1^{\circ}$. Since the chemistry of OTS SAMs is well developed and AIC of a-Si has been extensively studied in the semiconductor and MEMS industries, using a combination of such welldeveloped technologies to produce superhydrophobic surfaces has great potential in the MEMS industry.

\section{Acknowledgements}

This material is based on work supported by the US National Science Foundation under Grant Nos. CMMI-0600642 and CMMI-0645040. Any opinions, findings, and conclusions or recommendations expressed in this material are those of the author(s) and do not necessarily reflect the views of the National Science Foundation.

\section{References}

[1] Furstner, R.; Barthlott, W.; Neinhuis, C.; Walzel, P. Wetting and self-cleaning properties of artificial superhydrophobic surfaces. Langmuir 2005, 21, 956961.

[2] Zhu, L.; Xiu, Y.; Xu, J.; Hess, D.W.; Wong, C. P. Optimizing geometrical design of superhydrophobic surfaces for prevention of microelectromechanical system (MEMS) stiction. In Proceedings of the 56th Electronic Components and Technology Conference (ECTC), San Diego, USA, 30 May-2 June, 2006, pp. 1129-1135.

[3] Truesdell, R.; Mammoli, A.; Vorobieff, P.; van Swol, F.; Brinker, C.J. Drag reduction on a patterned superhydrophobic surface. Phys. Rev. Lett. 2006, 97, 044504.

[4] Barthlott, W.; Neinhuis, C. Purity of the sacred lotus, or escape from contamination in biological surfaces. Planta 1997, 202, 1-8.

[5] Nosonovsky, M.; Bhushan, B.Roughness-induced superhydrophobicity: A way to design non-adhesive surfaces. J. Phys.: Condens. Matter 2008, 20, 225009. 
[6] Li, J.; Xu, J.; Fan, L.; Wong, C. P. Lotus effect coating and its application for microelectromechanical systems stiction prevention. In Proceedings of the 54th Electronic Components and Technology Conference (ECTC), Las Vegas, USA, 1-4 June, 2004, pp. 943-947.

[7] Komvopoulos, K. Adhesion and friction force in microelectromechanical systems: Mechanisms, measurement, surface modification techniques, and adhesion theory. J. Adhes. Sci. Technol. 2003, 17, 477517.

[8] Maboudian, R.; Howe, R. T. Critical review: Adhesion in surface micromechanical structures. J. Vac. Sci. Technol. 1997, B15, 1-20.

[9] Tambe, N.; Bhushan, B. Nanotribological characterization of self-assembled monolayers deposited on silicon and aluminum substrates. Nanotechnology 2005, 16, 15491558.

[10] Feng, L.; Li, S. H.; Li, Y. H.; Li, H. J.; Zhang, L. J.; Zhai, J.; Song, Y. L.; Liu, B. Q.; Jing, L.; Zhu, D. B. Super-hydrophobic surfaces: From natural to artificial. Adv. Mater. 2002, 14, 1857-1860.

[11] Cao, L. L.; Price, T. P.; Weiss, M.; Gao, D. Super waterand oil-repellent surfaces on intrinsically hydrophilic and oleophilic porous silicon films. Langmuir 2008, 24, 16401643.

[12] Wu, X. F; Shi, G. Q. Production and characterization of stable superhydrophobic surfaces based on copper hydroxide nanoneedles mimicking the legs of water striders. J. Phys. Chem. B 2006, 110, 11247-11252.

[13] Liu, B.; He, Y. N.; Fan, Y.; Wang, X. G. Fabricating super-hydrophobic lotus-leaf-like surfaces through softlithographic imprinting. Macromol. Rapid Commun. 2006, 27, 1859-1864.

[14] Song, Y.; Zou, M. Superhydrophobic surfaces by dynamic nanomasking and deep reactive ion etching. Proc. IMechE, Part N: J. Nanoeng. Nanosystems 2007, 221, 41-48.

[15] Liu, H.; Ahmed, I.; Scherge, M. Microtribological properties of silicon and silicon coated with diamond like carbon, octadecyltrichlorosilane and stearic acid cadmium salt films: A comparative study. Thin Solid Films 2001, 381, 135-142.
[16] Ding, J.; Wong, P.; Yang, J. Friction and fracture properties of polysilicon coated with self-assembled monolayers. Wear 2006, 260, 209-214.

[17] Fu, Y.; Ding, J.; Yang, J. Investigation on the properties of anti-adhesive films on MEMS surfaces by AFM. Nongye Jixie Xuebao/Trans. Chin. Soc. Agric. Mach. 2006, 37, 182-184.

[18] Nast, O.; Hartmann, A. J. Influence of interface and Al structure on layer exchange during aluminum-induced crystallization of amorphous silicon. J. Appl. Phys. 2000, 88, 716-724.

[19] Schneider, J.; Heimburger, R.; Klein, J.; Muske, M.; Gall, S.; Fuhs, W. Aluminum-induced crystallization of amorphous silicon: Influence of temperature profiles. Thin Solid Films 2005, 487, 107-112.

[20] Qi, G. J.; Zhang, S.; Tang, T. T.; Li, J. F.; Sun, X. W.; Zeng, X. T. Experimental study of aluminum-induced crystallization of amorphous silicon thin films. Surf. Coat. Tech. 2005, 198, 300-303.

[21] Nast, O.; Brehme, S.; Pritchard, S.; Aberle, A. G.; Wenham, S. R. Aluminum-induced crystallization of silicon on glass for thin-film solar cells. Sol. Energy Mat. S. C. 2001, 65, 385-392.

[22] Zou, M.; Cai, L.; Brown, W. Nano-aluminum-induced low-temperature crystallization of PECVD amorphous silicon. Electrochem. Solid-State Lett. 2005, 8, G103G105.

[23] Gall, S.; Muske, M.; Sieber, I.; Nast, O.; Fuhs, W. Aluminum-induced crystallization of amorphous silicon. J. Non-Cryst. Solids 2002, 299, 741-745.

[24] Zou, M.; Cai, L.; Dorey, S. Silicon nanowires by aluminum-induced crystallization of amorphous silicon. Electrochem. Solid-State Lett. 2006, 9, G133-G135.

[25] Mirji, S. A. Octadecyltrichlorosilane adsorption kinetics on $\mathrm{Si}(100) / \mathrm{SiO}_{2}$ surfaces: Contact angle, AFM, FTIR and XPS analysis. Surf. Interface Anal. 2006, 38, 158-165.

[26] Wang, Y.; Lieberman, M. Growth of ultrasmooth octadecyltrichlorosilane self-assembled monolayers on $\mathrm{SiO}_{2}$. Langmuir 2003, 19, 1159-1167.

[27] Cassie, A .B. D.; Baxter, S. Wettability of porous surfaces. Trans. Faraday Soc. 1944, 40, 546-551. 